\title{
Anisotropy screening in Horndeski cosmologies
}

\author{
Alexei A. Starobinsky® ${ }^{1,2, *}$ Sergey V. Sushkov, ${ }^{2, \dagger}$ and Mikhail S. Volkov ${ }^{3,4,2, *}$ \\ ${ }^{1}$ L.D.Landau Institute for Theoretical Physics RAS, Moscow 119334, Russia \\ ${ }^{2}$ Department of General Relativity and Gravitation, Institute of Physics, Kazan Federal University, \\ 420008 Kazan, Russia \\ ${ }^{3}$ Institut Denis Poisson, UMR-CNRS 7013, Université de Tours, \\ Parc de Grandmont, 37200 Tours, France \\ ${ }^{4}$ Institute for Theoretical and Mathematical Physics, Lomonosov Moscow State University, \\ Leninskie Gory, GSP-1, 119991 Moscow, Russia
}

(Received 2 January 2020; accepted 26 February 2020; published 18 March 2020)

\begin{abstract}
We consider anisotropic cosmologies in a particular shift-symmetric Horndeski theory containing the $G^{\mu \nu} \partial_{\mu} \phi \partial_{\nu} \phi$ coupling, where $G^{\mu \nu}$ is the Einstein tensor. This theory admits stable in the future selfaccelerating cosmologies whose tensor perturbations propagate with the velocity very close to the speed of light such that the theory agrees with the gravity wave observations. Surprisingly, we find that the anisotropies within the Bianchi I homogeneous spacetime model are screened at early time by the scalar charge, whereas at late times they are damped in the usual way. Therefore, contrary to what one would normally expect, the early state of the universe in the theory cannot be anisotropic and (locally) homogeneous in the absence of spatial curvature. The early universe cannot be isotropic either, because it should then be unstable with respect to inhomogeneous perturbations. As a result, the early universe should be inhomogeneous. At the same time, we find that in the spatially curved Bianchi IX case the anisotropies can be strong at early times even in the presence of a scalar charge.
\end{abstract}

DOI: 10.1103/PhysRevD.101.064039

\section{INTRODUCTION}

It is usually assumed that the state of the universe close to the initial singularity should be strongly anisotropic $[1,2,3]$. This belief is based on the fact that spatial anisotropies produce in the Einstein equations terms proportional to the inverse square of the volume, $1 / V^{2}$, which become dominant when one goes backwards in time. In other words, anisotropic perturbations grow to the past. When the universe expands, the anisotropy terms decrease faster than the contribution of other forms of energy subject to the dominant energy condition and the universe rapidly approaches a locally isotropic state during inflation [4,5] (without the inflationary stage, this process may require a longtime or may not happen at all due to the possibility of a recollapse). Therefore, thinking about the early history of the universe, one could expect the isotropic phase of inflation to be generically preceded by an anisotropic

\footnotetext{
alstar@landau.ac.ru † sergey_sushkov@mail.ru

*volkov@lmpt.univ-tours.fr
}

Published by the American Physical Society under the terms of the Creative Commons Attribution 4.0 International license. Further distribution of this work must maintain attribution to the author(s) and the published article's title, journal citation, and DOI. Funded by SCOAP. phase. Although this argument seems quite robust, we shall present in what follows a peculiar cosmology whose anisotropies are damped at early times, hence the existence of a primary anisotropic phase is not as universal as one might think.

The theory we wish to discuss is the particular subset of the Horndeski theory for a gravitating scalar field [6] defined by the action (2.1) below. Its homogeneous and isotropic cosmologies were studied in $[7,8]$, but later it was discovered that theories of this type should be disfavored because they predict the speed of gravity waves (GW) different from the speed of light [9-11], whereas the recent GW170817 event shows that the GW speed is equal to the speed of light with very high precision [12]. However, this constraint applies rather to some solutions of the theory than to the theory itself. The theory admits stable in the future self-accelerating cosmologies whose tensor perturbations propagate with the velocity very close to the speed of light, the relative difference being proportional to $1 / \mathrm{V}$. Therefore, the theory can perfectly agree with the GW observation of [12] at late times, and we can extrapolate it to the early times as well since no observational data about the GW speed at redshifts $z>0.3$ are currently available.

We shall therefore study anisotropic cosmologies of the simplest Bianchi I homogeneous spacetime type within this Horndeski model. Surprisingly, we find that the 
anisotropies are screened at early times by the scalar charge, hence the standard argument in favor of strong anisotropies at early times does not always apply. However, the universe cannot be isotropic in this limit either, since it would then be unstable with respect to inhomogeneous perturbations. This suggests that the early universe should be inhomogeneous. At the same time, our numerics suggest that the universe can be strongly anisotropic close to the initial singularity within the Bianchi IX class, therefore the anisotropy screening is not generic for all Bianchi models.

\section{ISOTROPIC CASE}

To begin with, we summarize the essential properties of the isotropic solutions, some of which have never been discussed before. We consider the theory

$$
\begin{aligned}
S & =\frac{1}{2} \int\left(\mu R-\left(\alpha G_{\mu \nu}+\varepsilon g_{\mu \nu}\right) \nabla^{\mu} \phi \nabla^{\nu} \phi-2 \Lambda\right) \sqrt{-g} d^{4} x \\
& \equiv \frac{1}{2} \int L d^{4} x,
\end{aligned}
$$

where $\mu=M_{\mathrm{Pl}}^{2}$ is the Planck mass squared, the parameter $\alpha$ has the dimension of length squared, $[\alpha]=\left[L^{2}\right]=\left[M^{-2}\right]$ (we assume $c=\hbar=1$ ), the parameter $\varepsilon$ is dimensionless, while $\Lambda$ is related to the cosmological constant $\Lambda$ via $\Lambda=\mu \boldsymbol{\Lambda}$, one has $[\Lambda]=\left[L^{-4}\right]$. We consider the theory (2.1) as a classical theory of gravity valid for curvatures much less than the Planck curvature. No lower energy or curvature cutoff is required for its self-consistency, in particular for the absence of ghosts. Let us choose the spacetime metric as

$$
d s^{2}=-d t^{2}+\mathrm{a}^{2}(t)\left[d x_{1}^{2}+d x_{2}^{2}+d x_{3}^{2}\right],
$$

the case of more general homogeneous and isotropic metrics, including also an extra matter, was considered in [8]. Assuming the scalar field $\phi$ to depend only on time, the Friedmann equation for the Hubble parameter $H=\dot{\mathrm{a}} / \mathrm{a}$ is (see [8] for the explicit form of all equations in the theory)

$$
3 \mu H^{2}=\frac{1}{2}\left(\varepsilon-9 \alpha H^{2}\right) \dot{\phi}^{2}+\Lambda .
$$

The equation for the scalar $\phi$ can be integrated once to give

$$
\left(3 \alpha H^{2}-\varepsilon\right) \dot{\phi}=\frac{C}{\mathrm{a}^{3}},
$$

where the integration constants $C$ is the scalar charge associated with the invariance of the action under shifts $\phi \rightarrow \phi+\phi_{0}$. The notion of a scalar charge usually appears in the case of a complex scalar field, but we shall use it here to denote the amount of the real scalar field $\phi$ according to this definition.
If $C=0$ then one has either

$$
H^{2}=\frac{\Lambda}{3 \mu}, \quad \dot{\phi}=0
$$

or

$$
H^{2}=\frac{\varepsilon}{3 \alpha}, \quad \dot{\phi}^{2}=\frac{\Lambda}{\varepsilon}-\frac{\mu}{\alpha},
$$

in both cases the metric is pure de Sitter (all exact de Sitter solutions with $\dot{\phi} \neq 0$ in the generic scalar-tensor theory without a derivative coupling of the scalar field to gravity can be found in [13]).

If the charge $C$ does not vanish, then its effect should become negligible for $a \rightarrow \infty$, as seen from (2.4), hence the solutions should approach either (2.5) or (2.6) at late times (since $H^{2}$ and $\dot{\phi}^{2}$ should be positive, this imposes restrictions on values of the theory parameters.) If $C \neq 0$ then $\dot{\phi}$ can be algebraically expressed in terms of a, $H$. Using the values of the Hubble parameter and scale factor at present, $H_{0}, \mathrm{a}_{0}$, we introduce dimensionless variables $y=\left(H / H_{0}\right)^{2}$, $a=\mathrm{a} / \mathrm{a}_{0}$ and $\psi=\left(3 \alpha H_{0}^{2} \mathrm{a}_{0}^{3} / C\right) \dot{\phi}$, and also dimensionless parameters

$$
\Omega_{0}=\frac{\Lambda}{3 \mu H_{0}^{2}}, \quad \Omega_{6}=\frac{C^{2}}{18 \alpha \mathrm{a}_{0}^{6} H_{0}^{4} \mu}, \quad \zeta=\frac{\varepsilon}{3 \alpha H_{0}^{2}}
$$

(notice that the roman symbol a denotes the dimensionful scale factor, while $a$ stands for its dimensionless version). Equations (2.3), (2.4) then assume the form

$$
y=\Omega_{0}+\frac{\Omega_{6}[\zeta-3 y]}{a^{6}[\zeta-y]^{2}}, \quad \psi=\frac{1}{a^{3}(y-\zeta)},
$$

and since they should hold if $y=a=1$, it follows that

$$
\Omega_{6}=(\zeta-1)^{2} \frac{\left(1-\Omega_{0}\right)}{\zeta-3} .
$$

These equations determine $y(a)$ and $\psi(a)$, which determine $a(t)$ and $\phi(t)$.

Before considering solutions of the equations, let us study conditions for their stability. Considering small fluctuations $g_{\mu \nu} \rightarrow g_{\mu \nu}+\delta g_{\mu \nu}, \phi \rightarrow \phi+\delta \phi$, the metric perturbation can be decomposed into the scalar, vector, and tensor parts in the standard way [14], while $\delta \phi$ can be gauged away using the residual freedom of infinitesimal reparametrizations of the time coordinate, hence $\delta \phi=0$. The second variation of the action then splits into three independent parts describing the two tensor polarizations and the scalar mode (the vector sector contains no dynamics). Each of these parts has the structure 


$$
I=\frac{M_{\mathrm{Pl}}^{2}}{2} \int \mathrm{K}\left(\dot{X}^{2}-c_{s}^{2} \frac{P^{2}}{\mathrm{a}^{2}} X^{2}\right) \mathrm{a}^{3} d^{4} x
$$

$$
\mathrm{K}_{T}=1+\Omega_{6} \psi^{2}, \quad c_{T}^{2}=\frac{1-\Omega_{6} \psi^{2}}{1+\Omega_{6} \psi^{2}},
$$

where $P$ is the spatial momentum. In the case of tensor perturbations, $X$ is the tensor mode amplitude, while the kinetic term and the sound speed squared are with $\psi$ given by (2.8). In the scalar sector one has $X=$ $\delta g_{00}$ while

$$
\begin{aligned}
\mathrm{K}_{S} & =\frac{\left[\Omega_{6}+a^{6}(y-\zeta)^{2}\right]\left[\Omega_{6}(3 y+\zeta)-a^{6}(y-\zeta)^{3}\right]}{\left.a^{6} y(y-\zeta)^{2}\left[3 \Omega_{6}+a^{6}(y-\zeta)^{2}\right)\right]^{2}}, \\
c_{S}^{2} & =\frac{\left[3 \Omega_{6}+a^{6}(y-\zeta)^{2}\right]\left\{\left[a^{6}(y-\zeta)^{3}+\left(\Omega_{6} / 3\right)(13 y-3 \zeta)\right]^{2}-(52 / 9) \Omega_{6}^{2} y^{2}\right\}}{\left[\Omega_{6}+a^{6}(y-\zeta)^{2}\right]\left[\Omega_{6}(3 y+\zeta)-a^{6}(y-\zeta)^{3}\right]^{2}} .
\end{aligned}
$$

The functions $\mathrm{K}_{S}, \mathrm{~K}_{T}, c_{S}^{2}, c_{T}^{2}$ should be positive for the background solutions to be stable.

We can now analyze solutions of (2.8), and the simplest way to get them is to transform the first equation in (2.8) to

$$
a^{6}=\frac{\Omega_{6}(\zeta-3 y)}{(y-\zeta)^{2}\left(y-\Omega_{0}\right)} .
$$

Therefore, if $a \rightarrow 0$ then $y \rightarrow \zeta / 3$, while if $a \rightarrow \infty$ then either $y \rightarrow \Omega_{0}$ or $y \rightarrow \zeta$. In general, this defines three different solutions $y(a)$, but only one of them extends to the whole interval $a \in[0, \infty)$, the two others being unphysical [8]. One has to have $\zeta \sim \varepsilon / \alpha>0$, since otherwise $y(a)$ is not positive definite, yielding solutions with ghost [8]. Therefore, the parameters $\varepsilon$ and $\alpha$ should have the same sign. Let us assume first that $\alpha>0$ and $\varepsilon>0$, hence $\Omega_{6}>0$.

It is natural to assume that $0<\Omega_{0}<1$. Then the positivity of $\Omega_{6}$ defined by (2.9) requires that $\zeta>3$. In this case there exists only one solution $y(a)$ of (2.13), which fulfills

$$
\frac{\zeta}{3} \leftarrow y \rightarrow \Omega_{0} \quad \text { as } 0 \leftarrow a \rightarrow \infty .
$$

A direct verification reveals that $\mathrm{K}_{T}>0$ and $\mathrm{K}_{S}>0$ everywhere for this solution, hence the ghost is absent, while at large $a$ one has $c_{T}^{2}>0$ and $c_{S}^{2}>0$, hence the solution is free in this limit also from gradient instabilities. The profile of this solution is shown in Fig. 1.

The solution has two inflationary stages: an early inflation driven by the scalar $\phi$, with the Hubble rate $H^{2}=H_{0}^{2} y \approx H_{0}^{2} \zeta / 3=\varepsilon /(9 \alpha) \equiv H_{e}^{2}$, and a late inflation driven by the cosmological constant, with $H^{2} \approx H_{0}^{2} \Omega_{0}=$ $\Lambda /(3 \mu)=\boldsymbol{\Lambda} / 3 \equiv H_{l}^{2}$. Since $H_{l}$ is small, to have a hierarchy between the two inflationary scales, $H_{l} \ll H_{e}$, one should assume that $H_{\mathrm{e}} \sim \varepsilon / \alpha \sim \zeta$ is large, hence the coefficient $\alpha$ in (2.1) should be small. At the same time, one needs $H_{e}^{2} \ll M_{\mathrm{Pl}}^{2}=\mu$ for the classical theory to apply, hence $\alpha \gg 1 / \mu \equiv L_{\mathrm{Pl}}^{2}$. Therefore, even though $\alpha$ is small, it should be much larger than the Planck length squared. Similarly, although $\zeta$ is large, there is an upper bound $\zeta=\varepsilon /\left(3 \alpha H_{0}^{2}\right) \ll \mu / H_{0}^{2} \approx 10^{122}$.

One should say that, although the early inflationary stage is regular in terms of geometry, the scalar field is singular since one has $\psi \propto a^{-3} \propto e^{-3 H_{e} t}$ for $a \rightarrow 0$ and $t \rightarrow-\infty$. As a result, this is a kind of a "fast-roll" inflation, which makes questionable its usage for constructing viable cosmological models with approximately flat (scalefree) spectra of initial scalar and tensor perturbations generated by quantumgravitational effects. At the same time, as we shall see shortly, the universe should become inhomogeneous at early times, and that may change the way the spectra are derived. However, studying these issues goes beyond the scope of our purely classical analysis.

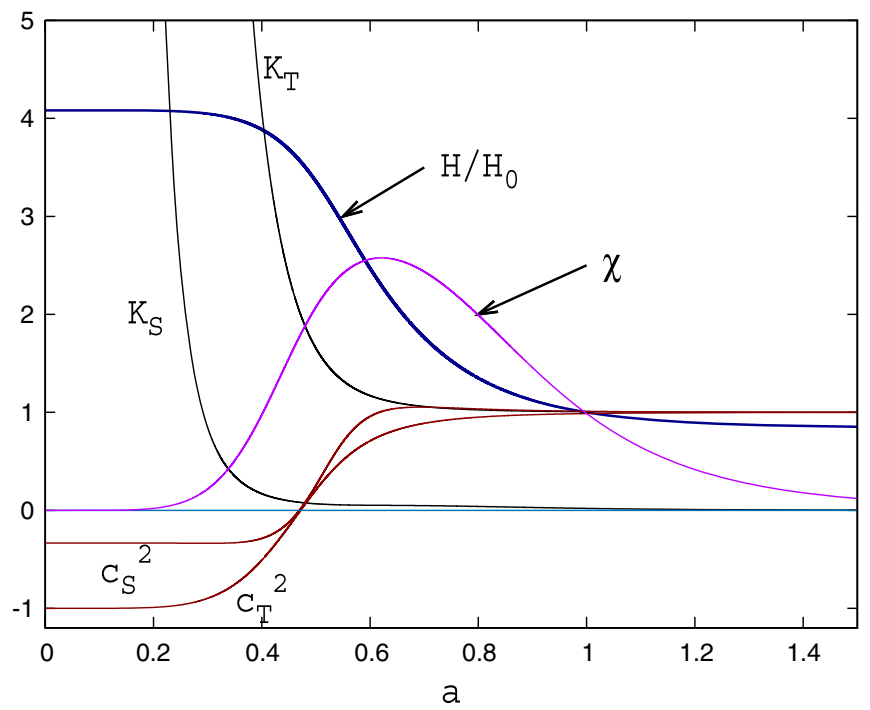

FIG. 1. Profile of $\sqrt{y}=H / H_{0}$ for $\Omega_{0}=0.7$ and $\zeta=50$. The kinetic terms $\mathrm{K}_{S}$ and $\mathrm{K}_{T}$ are always positive hence the ghost is absent. One has $\mathrm{K}_{T} \rightarrow 1$ while $\left.\mathrm{K}_{S} \propto 1 /\left(\zeta-\Omega_{0}\right) \Omega_{0} a^{6}\right)$ at large $a$. The sound speeds $\mathrm{c}_{S}^{2}$ and $\mathrm{c}_{T}^{2}$ approach unity at late times but become negative at small $a$, showing gradient instabilities with respect to inhomogeneous perturbations. The amplitude $\chi$ is the anisotropy defined by (3.13) (assuming that $\mathcal{B}=1$ ). 
As $a \rightarrow \infty$ one has $\mathrm{K}_{T}=1+\mathcal{O}\left(1 / a^{6}\right)$ hence the GW speed approaches the speed of light. At the same time, the present time moment corresponds to finite values $y=a=1$, when

$$
\mathrm{K}_{T}-1=\frac{1-\Omega_{0}}{\zeta-3} .
$$

Since $\zeta$ and $\Omega_{0}$ determine the Hubble rates of the early and late inflations, the ratio $\zeta / \Omega_{0}$ should be of the order of the early inflation energy scale divided by the late inflation energy scale, in which case $\mathrm{K}_{T}-1 \sim \Omega_{0} / \zeta \sim\left(H_{l} / H_{e}\right)^{2} \ll$ $10^{-15}$. This agrees with the observed bound on the relative difference between the GW velocity and the speed of light [12].

The solution develops gradient instabilities for small $a$ when $c_{T}^{2}$ and $c_{S}^{2}$ become negative because, as seen from (2.11) and (2.12), $c_{T}^{2} \rightarrow-1$ and $c_{S}^{2} \rightarrow-1 / 3$ as $a \rightarrow 0$. If $\zeta \gg 1$ then zeroes $c_{T}^{2}$ and $c_{S}^{2}$ are close to the point where $y=\zeta / 4$. Before discussing these instabilities, we shall consider below another property of the solution-the screening of anisotropies.

To finish this section, there are solutions with two inflationary stages also if $\alpha<0, \varepsilon<0$ and $\Omega_{6}<0$. However, in this case one has $0<\zeta<3$, and since $\zeta$ cannot be large, the difference $K_{T}-1$ is not small, hence these solutions give a wrong value for the GW speed.

\section{BIANCHI I-ANISOTROPY SCREENING}

Let us consider the Bianchi I metric

$$
d s^{2}=-N^{2} d t^{2}+\mathrm{a}_{1}^{2} d x_{1}^{2}+\mathrm{a}_{2}^{2} d x_{2}^{2}+\mathrm{a}_{3}^{2} d x_{3}^{2},
$$

where $N, \mathrm{a}_{k}$ and also $\phi$ are functions of $t$. Injecting to (2.1), the Lagrangian is

$L=-3 \mathrm{a}^{3}\left(\frac{2 \mu}{N}+\frac{\alpha \dot{\phi}^{2}}{N^{3}}\right) \mathcal{H}^{2}+\left(\frac{\epsilon \dot{\phi}^{2}}{N}-2 N \Lambda\right) \mathrm{a}_{1} \mathrm{a}_{2} \mathrm{a}_{3}$,

with

$3 \mathrm{a}^{3} \mathcal{H}^{2} \equiv \mathrm{a}_{1} \dot{\mathrm{a}}_{2} \dot{\mathrm{a}}_{3}+\mathrm{a}_{2} \dot{\mathrm{a}}_{1} \dot{\mathrm{a}}_{3}+\mathrm{a}_{3} \dot{\mathrm{a}}_{1} \dot{\mathrm{a}}_{2}=3 \mathrm{a}^{3}\left(\frac{\dot{\mathrm{a}}^{2}}{\mathrm{a}^{2}}-\dot{\beta}_{+}^{2}-\dot{\beta}_{-}^{2}\right)$,

where $\mathrm{a}_{1}=\mathrm{a} e^{\beta_{+}+\sqrt{3} \beta_{-}}, \mathrm{a}_{2}=\mathrm{a} e^{\beta_{+}-\sqrt{3} \beta_{-}}, \mathrm{a}_{3}=\mathrm{a} e^{-2 \beta_{+}}$. The field equations can be obtained by varying $L$ with respect to $N, \beta_{ \pm}, \phi$ and then setting $N=1$. This yields

$$
\begin{gathered}
3 \mu \mathcal{H}^{2}=\frac{1}{2}\left(\varepsilon-9 \alpha \mathcal{H}^{2}\right) \dot{\phi}^{2}+\Lambda, \\
\left(\sigma \mathrm{a}^{3} \dot{\beta}_{ \pm}\right)^{\cdot}=0, \\
\mathrm{a}^{3}\left(3 \alpha \mathcal{H}^{2}-\varepsilon\right) \dot{\phi}=C,
\end{gathered}
$$

with $\sigma=2 \mu+\alpha \dot{\phi}^{2}$, where $C$ is the scalar charge. If the anisotropies $\beta_{ \pm}$vanish, these equations reduce to (2.3), (2.4). If anisotropies do not vanish, then one has from (3.5)

$$
\dot{\beta}_{ \pm}=2 \mu \frac{\mathcal{B}_{ \pm}}{\sigma \mathrm{a}^{3}}
$$

where $\mathcal{B}_{ \pm}$are integration constants. Let us see what this implies first in the case when the scalar charge is zero, $C=0$. Then (3.6) can be solved by $\dot{\phi}=0$ while (3.4) and (3.7) yield

$$
\frac{\dot{\mathrm{a}}^{2}}{\mathrm{a}^{2}}=\dot{\beta}_{+}^{2}+\dot{\beta}_{-}^{2}+\frac{\Lambda}{3 \mu}, \quad \dot{\beta}_{ \pm}=\frac{\mathcal{B}_{ \pm}}{\mathrm{a}^{3}} .
$$

The anisotropy terms on the right in the first equation decay with time, hence anisotropy contribution becomes irrelevant and the universe rapidly approaches the isotropic de Sitter phase (2.5). However, the anisotropy terms become dominant at small a, when one can neglect the $\Lambda$-term and the universe is described by the Kasner metric for which $\mathrm{a}_{k} \propto t^{s_{k}}$ where the exponents $p_{k}$ are expressed in terms of $\mathcal{B}_{ \pm}$and fulfill $p_{1}+p_{2}+p_{3}=p_{1}^{2}+p_{2}^{2}+p_{3}^{2}=1$. This supports the standard view according to which anisotropies should be important close to the initial singularity.

If $C=0$ then (3.6) can be solved also by setting $3 \alpha \mathcal{H}^{2}-\varepsilon=0$, which gives

$$
\frac{\dot{\mathrm{a}}^{2}}{\mathrm{a}^{2}}=\dot{\beta}_{+}^{2}+\dot{\beta}_{-}^{2}+\frac{\varepsilon}{3 \alpha}, \quad \dot{\beta}_{ \pm}=\frac{2 \mu \varepsilon \mathcal{B}_{ \pm}}{(\mu \varepsilon+\alpha \Lambda) \mathrm{a}^{3}}, \quad \dot{\phi}^{2}=\frac{\Lambda}{\varepsilon}-\frac{\mu}{\alpha} .
$$

The solution approaches the isotropic de Sitter phase (2.6) at late times, but at early times the anisotropies are again dominant. However, unless if $\alpha \Lambda=\epsilon \mu$, this solution is unphysical because $\dot{\phi} \sim \psi$ approaches at late times a constant nonzero value, therefore, according to (2.11), the GW velocity is not equal to the speed of light.

Assume now that the scalar charge does not vanish, $C \neq 0$. Then one obtains from (3.6)

$$
\dot{\phi}=\frac{C}{\mathrm{a}^{3}\left(3 \alpha \mathcal{H}^{2}-\varepsilon\right)} .
$$

Injecting this to (3.4), setting $\mathcal{H}^{2}=H_{0}^{2} y$ and introducing the same $a, \psi, \Omega_{0}, \Omega_{6}, \zeta$ as above, one obtains exactly the same equations as in (2.8). Their solution for $y(a)$ and $\psi(a)$ is the same as the one described above and shown in Fig. 1. This time, however, it describes a Bianchi I spacetime with the anisotropies expressed by (3.7),

$$
\dot{\beta}_{ \pm}=\frac{\mathcal{B}_{ \pm}}{\mathrm{a}^{3}\left(1+\Omega_{6} \psi^{2}\right)},
$$


and with the Hubble rate

$\frac{\dot{\mathrm{a}}^{2}}{\mathrm{a}^{2}}=\dot{\beta}_{+}^{2}+\dot{\beta}_{-}^{2}+\mathcal{H}^{2}=\left(\frac{\dot{\beta}_{+}^{2}+\dot{\beta}_{-}^{2}}{\mathcal{H}^{2}}+1\right) \mathcal{H}^{2} \equiv H_{0}^{2}(\chi+1) y$.

Here

$$
\chi=\frac{\mathcal{B}}{a^{6}\left(1+\Omega_{6} \psi^{2}\right)^{2} y}
$$

is the relative contribution of the anisotropies to the total energy balance, its amplitude is $\mathcal{B}=\left(\mathcal{B}_{+}^{2}+\mathcal{B}_{-}^{2}\right) /\left(H_{0} a_{0}^{3}\right)^{2}$. Since the universe is highly isotropic at present, when $y=a=1$, one should assume that $\mathcal{B} \ll 1$, but this does not mean that isotropies have always been small.

Notice however that, according to (2.14), one has at early times

$y \approx \frac{\zeta}{3}, \quad \psi=\frac{1}{a^{3}(y-\zeta)} \propto a^{-3} \Rightarrow a^{3}\left(1+\Omega_{6} \psi^{2}\right) \propto a^{-3}$

and hence

$$
\dot{\beta}_{ \pm} \propto a^{3} \Rightarrow \dot{\beta}_{+}^{2}+\dot{\beta}_{-}^{2} \propto a^{6}
$$

As a result, the anisotropies tend to zero for $a \rightarrow 0$ and their contribution to the total energy balance is $\alpha a^{6}$ instead of $\propto 1 / a^{6}$. Therefore, the anisotropy effect is totally negligible at early times. This is true if only the scalar charge $C$ is nonzero, hence one can say that anisotropies are "screened by the scalar charge." Of course, the anisotropy contribution is suppressed at late time as well by the factor $1 / a^{6}$ in (3.12) (since $\psi \rightarrow 0$ as $a \rightarrow \infty$ ). As seen in Fig. 1, the anisotropy $\chi$ defined by (3.13) approaches zero at early and late times.

As there is no reason to assume the scalar charge to be zero, it follows that the anisotropies are screened at early times in our theory, at least in the Bianchi I case. This means that, unlike what one would normally expect, the state of the universe close to the singularity cannot be anisotropic and homogeneous. The early universe cannot be homogeneous and isotropic either, because then it would develop at small $a$ the gradient instability when the sound speed squared $c^{2}$ becomes negative, as seen in Fig. 1. A gradient (not ghost) instability indicates that the universe has a tendency to evolve to a different state. The instability is present both in the tensor and scalar sectors and it exists only for inhomogeneous perturbations, since the corresponding potential term in the effective action (2.10) contains the factor of $P^{2}$. In fact, the same condition that insures the anisotropy damping, $\Omega_{6} \psi^{2} \gg 1$, guarantees that $c_{T}^{2}$ and $c_{S}^{2}$ in (2.11) and (2.12) be negative. As a result, the early universe cannot be homogeneous and anisotropic, neither can it remain homogeneous and isotropic, hence it should evolve toward an inhomogeneous state.

The latter conclusion applies in fact to the whole stage of the primary inflation. As discussed above, when moving backward in time, the variable $y$ grows and the gradient instability starts at $y \approx \zeta / 4$, while the inflation starts only at $y \approx \zeta / 3$. Therefore, the primary inflation falls entirely within the instability region, hence it should be inhomogeneous. The consideration of an inhomogeneous inflation is beyond the scope of the present paper, but one may think that the spacetime geometry could then perhaps be described by something similar to the Gowdy metrics [15]. One might conjecture that the homogeneous component of the scalar charge will then be dispersed into its small-scale inhomogeneous fluctuations, so that the total spatially averaged value of $\Omega_{6} \psi^{2}$ does not exceed unity. However, an additional analysis is needed to study these issues.

One should also say that Eqs. (2.11), (2.12) describing the perturbations have been derived for the homogeneous and isotropic backgrounds. They can be used also in the anisotropic Bianchi I case at early times, since the anisotropies are then damped. However, at the intermediate times, when the anisotropies are not necessarily small, one should separately carry out the analysis of perturbations and rederive the coefficients $K_{T}, K_{S}, c_{T}^{2}, c_{S}^{2}$ by taking the anisotropies into account. This would probably give a different value of the GW speed $c_{T}^{2}$ (for example, taking the background inhomogeneities into account changes the GW speed [16]). However, the observational constraint on the GW speed apply only for relatively recent times, when the anisotropies should be small again, hence one can use in this case Eqs. (2.11), (2.12).

\section{BIANCHI IX CASE}

One may wonder if the anisotropy screening is typical only for the Bianchi I class or it occurs also for other Bianchi types. We shall therefore analyse the Bianchi IX class, in which case the spacetime metric is

$d s^{2}=-N^{2} d t^{2}+\frac{1}{4}\left(\mathrm{a}_{1}^{2} \omega_{1} \otimes \omega_{1}+\mathrm{a}_{2}^{2} \omega_{2} \otimes \omega_{2}+\mathrm{a}_{3}^{2} \omega_{3} \otimes \omega_{3}\right)$,

where $\omega_{a}$ are the invariant forms on $S^{3}$ subject to $d \omega_{a}+\epsilon_{a b c} \omega_{b} \wedge \omega_{c}=0$, while $\mathrm{a}_{k}$ and the scalar $\phi$ depends only on time. The Lagrangian in (3.2) generalizes to

$$
\begin{aligned}
8 L= & \frac{6 \mu \mathrm{a}^{3}}{N}\left(\frac{\mathcal{K} N^{2}}{\mathrm{a}^{2}}-\mathcal{H}^{2}\right)-\frac{3 \alpha \mathrm{a}^{3}}{N^{3}} \dot{\phi}^{2}\left(\mathcal{H}^{2}+\frac{\mathcal{K} N^{2}}{\mathrm{a}^{2}}\right) \\
& +\left(\frac{\varepsilon}{N} \dot{\phi}^{2}-2 N \Lambda\right) \mathrm{a}^{3},
\end{aligned}
$$


where $\mathcal{H}$ is the same as in (3.3), while the anisotropy potential is

$$
\mathcal{K}=-\frac{1}{3} e^{-8 \beta_{+}}\left(4 e^{6 \beta_{+}} \cosh ^{2}\left(\sqrt{3} \beta_{-}\right)-1\right)\left(4 e^{6 \beta_{+}} \sinh ^{2}\left(\sqrt{3} \beta_{-}\right)-1\right) .
$$

Varying the Lagrangian and setting $N=1$ gives the equations (with $\sigma=2 \mu+\alpha \dot{\phi}^{2}$ )

$$
\begin{gathered}
3 \mu\left(\mathcal{H}^{2}+\frac{\mathcal{K}}{\mathrm{a}^{2}}\right)+\frac{3}{2} \alpha \dot{\phi}^{2}\left(3 \mathcal{H}^{2}+\frac{\mathcal{K}}{\mathrm{a}^{2}}\right)=\frac{\varepsilon}{2} \dot{\phi}^{2}+\Lambda, \\
\frac{1}{\mathrm{a}^{2}}(\sigma \mathrm{a} \dot{a})^{\circ}=\sigma\left(\frac{1}{2} \mathcal{H}^{2}-\dot{\beta}_{+}^{2}-\dot{\beta}_{-}^{2}\right)+\left(\frac{\alpha}{2} \dot{\phi}^{2}-\mu\right) \frac{\mathcal{K}}{\mathrm{a}^{2}}-\frac{\varepsilon}{2} \dot{\phi}^{2}+\Lambda, \\
\left(\sigma \mathrm{a}^{3} \dot{\beta}_{ \pm}\right)^{\cdot}=\mathrm{a}\left(\mu-\frac{\alpha}{2} \dot{\phi}^{2}\right) \frac{\partial \mathcal{K}}{\partial \beta_{ \pm}}, \\
\mathrm{a}^{3}\left(3 \alpha\left(\mathcal{H}^{2}+\frac{\mathcal{K}}{\mathrm{a}^{2}}\right)-\varepsilon\right) \dot{\phi}=C,
\end{gathered}
$$

where the first equation (4.4) is actually the first integral of the remaining (4.5)-(4.7). The effect of anisotropies is encoded in Eqs. (4.4), (4.7) only through the term $\mathcal{K} \geq 1$. Therefore, applying the same transformations as before, one obtains instead of (2.8) the equations

$$
y=\Omega_{0}+\frac{\Omega_{2}}{a^{2}}+\frac{\Omega_{6}\left[\zeta-3 y+\Omega_{2} / a^{2}\right]}{a^{6}\left[\zeta-y+\Omega_{2} / a^{2}\right]^{2}}, \quad \psi=\frac{1}{a\left[a^{2}(\zeta-y)+\Omega_{2}\right]} .
$$

Although they resemble Eqs. (2.8), they do not form a closed system since they contain $\Omega_{2}=-\mathcal{K} /\left(H_{0}^{2} \mathrm{a}_{0}^{2}\right)$ where $\mathcal{K}$ is the function of the anisotropies defined by (4.3). However, the system of equations becomes closed in the isotropic case, when one can consistently set $\beta_{ \pm}=0$, which yields $\mathcal{K}=1$ and $\Omega_{2}=-1 /\left(H_{0}^{2} \mathrm{a}_{0}^{2}\right)$. The solutions for $y(a)$ then can be obtained from (4.8) by applying the Cardano formula, and they are such that $y(a)$ approaches $\Omega_{0}$ as $a \rightarrow \infty$ but vanishes at $a=a_{\min }>0$ and becomes negative for $a<a_{\min }$ [8]. These solutions describe bouncing universes which shrink from infinity to the minimal size $a_{\text {min }}$ when the Hubble parameter $H=H_{0} \sqrt{y}$ vanishes, and then expand again [8]. Such a bouncing behavior is due to the positive spatial curvature. These bounces exist for any however small value of $\Omega_{0}>0$. If $C=0$ then $\Omega_{6}=0$ and (2.8) reduce to

$$
y=\Omega_{0}+\frac{\Omega_{2}}{a^{2}}, \quad \psi=\frac{1}{\left(\zeta-\Omega_{0}\right) a^{3}},
$$

and using $y=(\dot{a} / a)^{2} / H_{0}^{2}$ yields

$$
a(t)=\sqrt{\frac{3 \mu}{\Lambda \mathrm{a}_{0}^{2}}} \cosh \left(\sqrt{\frac{\Lambda}{3 \mu}} t\right) .
$$

This describes the de Sitter metric expressed in coordinates with compact spatial sections.
These bounces can be generalized to include small anisotropies, because expanding the equations (4.6) for $\beta_{ \pm}$up to the fist order yields $\left(\sigma \mathrm{a}^{3} \dot{\beta}_{ \pm}\right)^{\circ}=0$ and hence $\dot{\beta}_{ \pm}=2 \mu \mathcal{B}_{ \pm} /\left(\sigma \mathrm{a}^{3}\right)$. Since the value of $\sigma \mathrm{a}^{3}$ is bounded below for a bounce, it follows that if the integration constants $\mathcal{B}_{ \pm}$are small, the anisotropies always remain small and only produce a small correction to the Hubble rate. The zero of $H$ shifts slightly due to the anisotropies, since one has

$$
H^{2}=\dot{\beta}_{+}^{2}+\dot{\beta}_{-}^{2}+H_{0}^{2} y
$$

Therefore, if $\beta_{ \pm}=0$ then $H$ vanishes at $a_{\min }$ where $y$ vanishes, but if $\dot{\beta}_{ \pm} \neq 0$ then the zero of $H$ shifts to the region $a<a_{\min }$ where $y<0$.

Now, if $C=\dot{\phi}=0$ then the equations also admit the slightly anisotropic bounces, but they admit as well strongly anisotropic solutions with initial singularity. In other words, increasing the amplitude of anisotropies shifts the bounce position more and more until it reaches $a=0$, after which the solutions are no longer bounces and show the initial curvature singularity. Equation (4.4) then reduces to

$$
\frac{\dot{\mathrm{a}}^{2}}{\mathrm{a}^{2}}=\dot{\beta}_{-}^{2}+\dot{\beta}_{+}^{2}-\frac{\mathcal{K}}{\mathrm{a}^{2}}+\frac{\Lambda}{3 \mu},
$$


where the positive anisotropy terms on the right are large enough to overcome the negative term, thereby eliminating the bouncing behavior. The solutions are then characterized by a sequence of "Kasner epochs" during which $\partial \mathcal{K} / \partial \beta_{ \pm} \approx 0$ and the universe is approximately described by the Kasner metric with $\mathrm{a}^{3} \dot{\beta}_{ \pm} \equiv \mathcal{B}_{ \pm} \approx$ const. The term $\partial \mathcal{K} / \partial \beta_{ \pm}$becomes important only during short moments when $\mathcal{B}_{ \pm}$change, after which the next Kasner epoch with new values of $\mathcal{B}_{ \pm}$starts [1].

One can wonder if a similar evolution near singularity is possible also when the scalar charge $C$ does not vanish? It seems at first that the answer should be negative, since the Kasner epochs during which the solution is approximately Bianchi I seem to be forbidden by the anisotropy screening.

To clarify the situation, we solved numerically the system of second order equations (4.5), (4.6) together with the equation obtained by differentiating (4.7). The first order equation (4.4) was used to constraint the initial values, and we checked that the constraint propagates. We also checked that the scalar charge defined by (4.7) remains constant during the evolution, as it should. We chose the initial data to describe a slightly anisotropic universe of a finite size and then integrated the equations to the past. It turns out that if the initial anisotropy is small, then the scale factor $\mathrm{a}(t)$ first decreases to the past, then passes through a minimal nonzero value, and then stars increasing. The solution is of the bounce type and the anisotropies always remain small. However, if the initial anisotropy is large enough, then the scale factor always decreases to the past while the anisotropies grow. The singularity is strongly anisotropic.

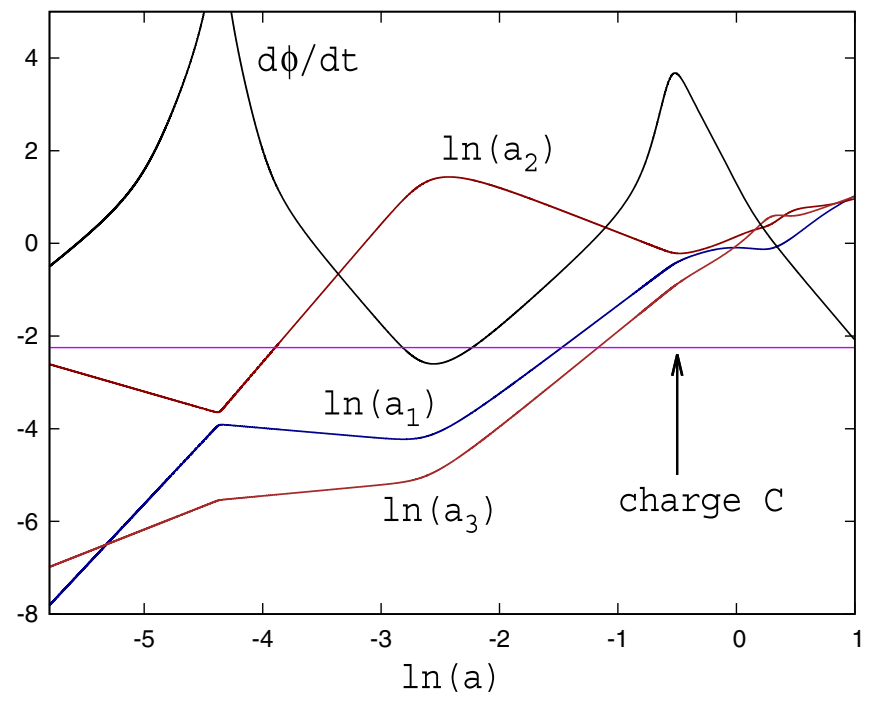

FIG. 2. Solution of Eqs. (4.4)-(4.7) for $\mu=\Lambda=\varepsilon=1, \alpha=$ 0.1 and with the initial values $\mathrm{a}=5, \dot{\beta}_{ \pm}=0, \beta_{+}=0, \beta_{-}=0.05$, $\dot{\phi}=0.02$ (assuming all parameters to be dimensionless and expressed in Planck units). The solution shows a sequence of Kasner epochs during which $\ln \left(\mathrm{a}_{k}\right) / \ln (\mathrm{a}) \approx$ const. The scalar $\dot{\phi}$ oscillates but the charge $C$ is constant.
A typical solution is shown in Fig. 2. Surprisingly, it demonstrates a sequence of Kasner epochs during which it must approach the Bianchi I regime. This seems to contradict the fact that the anisotropies should then be screened. However, the explanation is the following. In the Bianchi I case the anisotropies are screened because $\psi \propto a^{-3}$, which makes large the denominator in (3.11). In the Bianchi IX case Eq. (4.8) yields $y=\Omega_{2} /\left(3 a^{2}\right)+\mathcal{O}(1)$ and $\psi=$ $3 /\left(2 \Omega_{2} a\right)+\mathcal{O}(1)$ for small $a$. Since $\Omega_{2} \sim \mathcal{K}$ one has $\psi \propto 1 /(\mathcal{K} a)$, and since $\mathcal{K} \geq 1$, it follows that $\psi$ grows not faster than $a^{-1}$. This implies that the denominator in (3.11) behaves as $a / \mathcal{K}^{2}$ and tends to zero, hence $\dot{\beta}_{ \pm}$ expressed by (3.11) are large. Therefore, the anisotropies are not screened in the Bianchi IX case, hence their screening is not a generic feature for all Bianchi models. A more detailed analysis is needed to find out if the solutions can be chaotic [1]. The stability of these solutions with respect to inhomogeneous perturbations remains an open issue to study.

\section{CONCLUSION}

We studied anisotropic cosmologies in the shift-symmetric, nonminimally coupled Horndeski model (2.1). Even though this model is thought to be disfavored by the GW observations, its homogeneous and isotropic solution propagates tensor perturbations with the velocity that can be insensitively close to the speed of light. Surprisingly, it turns out that the spatial anisotropies in this theory get damped at early times in the Bianchi I case, instead of being amplified. Therefore, the standard argument in favor of strong anisotropies at early times does not always apply. However, it seems that the anisotropy screening is not generic for all Bianchi types, since our numerics suggest that the universe can be strongly anisotropic close to the initial singularity within the Bianchi IX class, where positive spatial curvature is present. The scale factor $a(t)$ and the scalar field $\phi(t)$ can also show a regular bouncing behavior in this case, similarly to the minimally coupled scalar field in the closed Friedmann-Lemaitre-Robertson-Walker spacetime [17], but the generic solution contains a curvature singularity.

Although the anisotropies are screened at early times in the Bianchi I case, the universe does not approach an homogeneous and isotropic state, since it would then be unstable with respect to inhomogeneous perturbations. This suggests that the early stage of the universe should be essentially inhomogeneous. To study such inhomogeneous cosmologies requires a separate analysis.

To the best of our knowledge, a similar systematic analysis of anisotropic cosmologies for generic Horndeski 
models has never been carried out before, although anisotropic cosmologies with scalars have been studied. For example, anisotropies in the theory with a conformally coupled scalar field [18] and also in the $R+R^{2}$ gravity $[19,20]$ have been discussed, both cases being conformally dual to the ordinary gravity with a scalar field. But in our case the theory cannot be conformally transformed to the Einstein frame. As a result, qualitatively new behavior of anisotropy takes place.

\section{ACKNOWLEDGMENTS}

The work of A. A. S. and S. V.S was supported by the Russian Foundation for Basic Research, Grant No. 19-5215008. The work of M. S. V. was partly supported by the French National Center of Scientific Research within the collaborative French-Russian research program, Grant No. 289860, as well as by the Russian Government Program of Competitive Growth of the Kazan Federal University.
[1] V. A. Belinsky, I. M. Khalatnikov, and E. M. Lifshitz, Adv. Phys. 19, 525 (1970).

[2] C. B. Collins and S. W. Hawking, Astrophys. J. 180, 317 (1973).

[3] V. A. Belinsky, I. M. Khalatnikov, and E. M. Lifshitz, Adv. Phys. 31, 639 (1982).

[4] A. A. Starobinsky, JETP Lett. 37, 66 (1983).

[5] R. M. Wald, Phys. Rev. D 28, 2118 (1983).

[6] G. W. Horndeski, Int. J. Theor. Phys. 10, 363 (1974).

[7] S. V. Sushkov, Phys. Rev. D 80, 103505 (2009).

[8] A. A. Starobinsky, S. V. Sushkov, and M. S. Volkov, J. Cosmol. Astropart. Phys. 06 (2016) 007.

[9] P. Creminelli and F. Vernizzi, Phys. Rev. Lett. 119, 251302 (2017).

[10] J. M. Ezquiaga and M. Zumalacarregui, Phys. Rev. Lett. 119, 251304 (2017).

[11] T. Baker, E. Bellini, P. G. Ferreira, M. Lagos, J. Noller, and I. Sawicki, Phys. Rev. Lett. 119, 251301 (2017).
[12] B. Abbott et al. (LIGO Scientific, Virgo Collaborations), Phys. Rev. Lett. 119, 161101 (2017).

[13] H. Motohashi and A. A. Starobinsky, J. Cosmol. Astropart. Phys. 11 (2019) 025.

[14] S. Weinberg, Cosmology, (Oxford University Press, New York, 2008)

[15] R. H. Gowdy, Ann. Phys. (N.Y.) 83, 203 (1974).

[16] E. J. Copeland, M. Kopp, A. Padilla, P. M. Saffin, and C. Skordis, Phys. Rev. Lett. 122, 061301 (2019).

[17] A. A. Starobinsky, Sov. Astron. Lett. 4, 82 (1978).

[18] A. Y. Kamenshchik, E. O. Pozdeeva, S. Y. Vernov, A. A. Starobinsky, A. Tronconi, and G. Venturi, Phys. Rev. D 97, 023536 (2018).

[19] V. T. Gurovich and A. A. Starobinsky, Sov. Phys. JETP 50, 844 (1979).

[20] D. Muller, A. Ricciardone, A. A. Starobinsky, and A. Toporensky, Eur. Phys. J. C 78, 311 (2018). 